\title{
SHORT CHAINS AND SHORT CYCLES OF MODULES
}

\author{
I. REITEN, A. SKOWROŃSKI, AND S. O. SMALØ
}

(Communicated by Maurice Auslander)

\begin{abstract}
We show that for a large class of artin algebras including the algebras of finite representation type, an indecomposable module $M$ is not the middle of a short chain if and only if there is no short cycle $M \rightarrow N \rightarrow M$ of nonzero nonisomorphisms between indecomposable modules. We apply this to get sufficient conditions for modules to be determined by their composition factors. We also show that if for an algebra of finite representation type there is a sincere indecomposable $\Lambda$-module that is not the middle of a short chain, then $\Lambda$ is a tilted algebra.
\end{abstract}

\section{INTRODUCTION}

Let $\Lambda$ be an artin algebra over a commutative artin ring $R$. A (finitely generated) $\Lambda$-module $C$ is said to be the middle of a short chain if there is some indecomposable $\Lambda$-module $X$ with $\operatorname{Hom}_{\Lambda}(X, C) \neq 0$ and $\operatorname{Hom}_{\Lambda}(C, D T r X) \neq 0$. Indecomposable modules that are not the middle of a short chain were introduced and studied in [1]. For example it was shown that if $B$ and $C$ are indecomposable modules that are not the middle of a short chain and $B$ and $C$ have the same composition factors, then $B$ and $C$ are isomorphic. Also it was shown in [1] that if $\Lambda$ is an algebra of finite representation type and $R=k$ is an algebraically closed field, then there is always a finite covering of $\Lambda$ such that no indecomposable module is the middle of a short chain. This indicates that large cycles are better behaved than small cycles. In fact, we shall here show that being the middle of a short chain is closely related to being (on what we call) a short cycle: An indecomposable $\Lambda$-module $M$ is on a short cycle if there exists an indecomposable $\Lambda$-module $N$ and nonzero nonisomorphisms $f: M \rightarrow N$ and $g: N \rightarrow M$. We show that the concepts are the same if $\Lambda$ is of finite representation type, or more generally, if $\Lambda$ has no $A_{\infty}^{\infty}$-sectional chains.

Received by the editors March 18, 1991 and, in revised form, June 20, 1991. The contents of this paper were presented at the Oberwolfach meeting on Representation Theory of Algebras, May 1991, at the Warwick meeting on Ring Theory and its Applications, July 1991, and at the RIMS Symposium Representation Theory of Finite Groups and Finite Dimensional Algebras, Kyoto, October 1991.

1991 Mathematics Subject Classification. Primary 16G10, 16G70, 16D90; Secondary 16E10.

Key words and phrases. Short chains, short cycles, tilted algebras.

This research was partially supported by the Norwegian Research Council of Science and Humanities. This work was done while the second author visited Trondheim. He was partially supported by Polish Ministery of Education under Research Project R.P.I. 10. 
We say that a chain $\cdots \rightarrow C_{i} \rightarrow C_{i+1} \rightarrow \cdots$ with $i \in Z$ of irreducible maps between indecomposable modules is $A_{\infty}^{\infty}$-sectional if $D \operatorname{Tr} C_{i+1} \not C_{i-1}$ for all $i$. This is the content of $\S 1$.

In $\S 2$ we apply the results from $\S 1$ to prove that if $\Lambda$ has no $A_{\infty}^{\infty}$-sectional chains and $M$ and $N$ are indecomposable modules with the same composition factors, with $M$ not being the middle of a short chain, then $M$ and $N$ are isomorphic.

In $\S \S 3$ and 4 we investigate algebras having a sincere module not being the middle of a short chain and compare with algebras having sincere directing modules. In $\S 3$ we give some necessary conditions on $\Lambda$, like having global dimension at most two and having no oriented cycle in the ordinary quiver. In $\S 4$ we show that if $\Lambda$ is, in addition, of finite representation type, then $\Lambda$ is a tilted algebra.

\section{SHORT CHAINS AND SHORT CYCLES}

We say that a chain of two nonzero maps $X \rightarrow M \rightarrow D \operatorname{Tr} X$ between $\Lambda$ modules with $X$ indecomposable is a short chain, and $M$ is said to be the middle of a short chain (see [1]). For indecomposable modules $M$ and $N$ we shall call a chain of two nonzero nonisomorphisms $M \rightarrow N \rightarrow M$ a short cycle and say that in this case $M$ and $N$ lie on a short cycle. We shall show that if an indecomposable module $M$ is the middle of a short chain, then it lies on a short cycle, and we shall show that the converse holds if the algebra $\Lambda$ has no $A_{\infty}^{\infty}$-sectional chains. We say that a chain of irreducible maps between indecomposable modules $\cdots X_{i-1} \rightarrow X_{i} \rightarrow X_{i+1} \rightarrow \cdots,-m<i<n$, is sectional if $D T r X_{i+1} \not X_{i-1}$ for all $-m<i<n$, and it is $A_{\infty}^{\infty}$-sectional if $n=m=\infty$. Before we state the main result, we start out with some preliminary properties. Recall that the $\operatorname{radical} \operatorname{rad}(A, B)$ of $A$ and $B$ in $\bmod \Lambda$ is the set of maps $f: A \rightarrow B$ where $q f p$ is a nonisomorphism for all nonzero maps $p$ and $q$. We will often denote $\operatorname{Hom}_{\Lambda}(A, B)$ by $(A, B)$.

Lemma 1.1. Let $M$ be a $\Lambda$-module that is not the middle of a short chain, and let $f: A \rightarrow B$ be an irreducible map between indecomposable modules.

If $(M, A) \neq 0$ and $(B, M) \neq 0$, then $\operatorname{rad}(M, B) \neq 0$ and $\operatorname{rad}(A, M) \neq 0$. Proof. Let $M$ be a $\Lambda$-module that is not the middle of a short chain, and let $f: A \rightarrow B$ be an irreducible map between indecomposable modules. Assume that $(M, A) \neq 0$ and $(B, M) \neq 0$. Since $M$ is not the middle of a short chain, we have $(M, D \operatorname{Tr} B)=0$. Consider the exact sequence $0 \rightarrow D \operatorname{Tr} B \rightarrow$ $E \rightarrow^{g} B$, where $g: E \rightarrow B$ is minimal right almost split. We then get an exact sequence $0 \rightarrow(M, E) \rightarrow \operatorname{rad}(M, B)$. Since $f: A \rightarrow B$ is irreducible, $A$ is a summand of $E$. Then $(M, A) \neq 0$ implies $(M, E) \neq 0$, which again implies $\operatorname{rad}(M, B) \neq 0$. Similarly we prove that $\operatorname{rad}(A, M) \neq 0$.

Lemma 1.2. Let $(n, m) \in(\mathbf{N} \cup\{\infty\}) \times(\mathbf{N} \cup\{\infty\})$ and let $\cdots \rightarrow X_{i-1} \rightarrow X_{i} \rightarrow \cdots$ be a chain of irreducible maps between indecomposable modules $X_{i}$ for $-m-1<$ $i<n$. Let $M$ be a $\Lambda$-module that is not the middle of a short chain such that $\left(M, X_{i}\right) \neq 0$ for $i<0$ and $\left(X_{i}, M\right) \neq 0$ for $i \geq 0$. Then $\operatorname{rad}\left(M, X_{j}\right) \neq 0$ for $-m<j<n$ and $\operatorname{rad}\left(X_{j}, M\right) \neq 0$ for $-m-1<j<n-1$.

Proof. Let $(n, m) \in(\mathbf{N} \cup\{\infty\}) \times(\mathbf{N} \cup\{\infty\})$, let $\cdots \rightarrow X_{i} \rightarrow X_{i+1} \rightarrow \cdots$ be a chain of irreducible maps between indecomposable modules $X_{i}$ for $-m-1<$ 
$i<n$, and let $M$ be a $\Lambda$-module that is not the middle of a short chain with $\left(M, X_{i}\right) \neq 0$ for $-m-1<i<0$ and $\left(X_{i}, M\right) \neq 0$ for $0 \leq i<n$. Then we use Lemma 1.1 on the irreducible morphism $X_{-1} \rightarrow X_{0}$ to obtain that $\operatorname{rad}\left(M, X_{0}\right) \neq 0$ and $\operatorname{rad}\left(X_{-1}, M\right) \neq 0$. Hence $\left(X_{-1}, M\right) \neq 0$, and by induction we get that $\operatorname{rad}\left(X_{i}, M\right) \neq 0$ and $\operatorname{rad}\left(M, X_{i+1}\right) \neq 0$ for $-m-1<$ $i<0$. We also have that $\operatorname{rad}\left(M, X_{0}\right) \neq 0$ implies that $\left(M, X_{0}\right) \neq 0$, and hence by induction in the appropriate direction we get that $\operatorname{rad}\left(M, X_{i}\right) \neq 0$ and $\operatorname{rad}\left(X_{i-1}, M\right) \neq 0$ for $0 \leq i<n$. Hence the result follows.

Lemma 1.3. Let $(n, m) \in(\mathbf{N} \cup\{\infty\}) \times(\mathbf{N} \cup\{\infty\})$ and let $\cdots \rightarrow X_{-i} \rightarrow \cdots X_{0} \rightarrow$ $\cdots \rightarrow X_{i} \rightarrow \cdots$ be a chain of irreducible maps between indecomposable modules $X_{i}$ with $-m<i<n$ such that $\left(M, X_{i}\right) \neq 0$ and $\left(X_{i}, M\right) \neq 0$ for all $i$.

If $M$ is not the middle of a short chain then the above chain is sectional.

Proof. With the above assumptions, if the chain was not sectional, there would be some $i$ with $D T r X_{i+1} \simeq X_{i-1}$. But since $\left(X_{i+1}, M\right) \neq 0$ and $\left(M, X_{i-1}\right) \neq$ $0, M$ would then be the middle of a short chain.

Lemma 1.4. Let $M$ and $N$ be indecomposable modules with $\operatorname{rad}(M, N) \neq$ 0 . Then there is either an infinite chain $\cdots X_{j} \rightarrow \cdots \rightarrow X_{-1} \rightarrow X_{0}=N$ of irreducible maps between indecomposable modules with $\left(M, X_{j}\right) \neq 0$ for all $j \leq 0$ or a finite chain $M=X_{i} \rightarrow \cdots \rightarrow X_{-1} \rightarrow X_{0}=N$ of irreducible maps between indecomposable modules with nonzero composition.

Proof. Assume that there is no finite chain of irreducible maps between indecomposable modules $M \rightarrow \cdots \rightarrow N$, with nonzero composition. Assume that $\operatorname{rad}(M, N) \neq 0$, and let $f: M \rightarrow N$ be nonzero and not an isomorphism. Let $h: E \rightarrow N$ be a minimal right almost split map. Since $f$ is not an isomorphism, it factors through $h: E \rightarrow N$. Hence there is some indecomposable summand $E_{1}$ of $E$ such that the induced composition $M \rightarrow E_{1} \rightarrow N$ is nonzero. Since $E_{1} \rightarrow N$ is irreducible, $M \rightarrow E_{1}$ is not an isomorphism by our assumption. Continuing this way, we get an infinite chain $\cdots \rightarrow X_{j} \rightarrow \cdots X_{-1} \rightarrow X_{0}=N$ of irreducible maps between indecomposable modules, with $\left(M, X_{j}\right) \neq 0$ for all $j \leq 0$. This finishes the proof.

We can now put the lemmas together to get

Proposition 1.5. Let $M$ and $N$ be indecomposable $\Lambda$-modules, and assume that $M$ is not the middle of a short chain.

If $\operatorname{rad}(M, N) \neq 0$ and $\operatorname{rad}(N, M) \neq 0$, then $N$ is on an $A_{\infty}^{\infty}$-sectional chain.

Proof. Let $M$ and $N$ be indecomposable modules with $M$ not the middle of a short chain, and assume $\operatorname{rad}(M, N) \neq 0$ and $\operatorname{rad}(N, M) \neq 0$.

We first want to show that there is an infinite chain of irreducible maps between indecomposable modules $\cdots \rightarrow X_{i} \rightarrow \cdots X_{-1} \rightarrow X_{0}=N$ with $\left(M, X_{j}\right) \neq$ 0 for $j \leq 0$. By Lemma 1.4 we can assume, since $\operatorname{rad}(M, N) \neq 0$, that we have a finite chain $M=X_{i} \rightarrow \cdots X_{0}=N$ of irreducible maps with nonzero composition, and hence $\left(M, X_{j}\right) \neq 0$ for $i \leq j \leq 0$. Using that $(N, M) \neq 0$, we get $\operatorname{rad}(M, M) \neq 0$ by Lemma 1.2 since $M$ is not the middle of a short chain. If there was a finite chain of irreducible maps between indecomposable modules $M=Y_{n} \rightarrow \cdots \rightarrow Y_{-1} \rightarrow Y_{0}=M$ with nonzero composition, then $\left(M, Y_{j}\right) \neq 0$ and $\left(Y_{j}, M\right) \neq 0$ for $n \leq j \leq 0$. But then we would have a 
sectional cycle by Lemma 1.3, which is impossible by [5]. Hence by Lemma 1.4 there would be an infinite chain of irreducible maps between indecomposable modules $\cdots \rightarrow Y_{j} \rightarrow \cdots \rightarrow Y_{-1} \rightarrow Y_{0}=M$ with $\left(M, Y_{j}\right) \neq 0$ for $j \leq 0$. Writing $Y_{t}=X_{i+t}$ for $t<0$, we get an infinite chain of irreducible maps between indecomposable modules $\cdots \rightarrow X_{j-1} \rightarrow X_{j} \rightarrow \cdots \rightarrow X_{-1} \rightarrow X_{0}=N$ with $\left(M, X_{j}\right) \neq 0$ for all $j \leq 0$.

By duality we get an infinite chain of irreducible maps between indecomposable modules $N=X_{0} \rightarrow X_{1} \rightarrow X_{2} \rightarrow \cdots \rightarrow X_{j} \rightarrow \cdots$ with $\left(X_{j}, M\right) \neq 0$ for all $j \geq 0$. Since then $\left(X_{j}, M\right) \neq 0$ and $\left(M, X_{j}\right) \neq 0$ for all $j$ by Lemma 1.2 , it follows by Lemma 1.3 that the chain

$$
\cdots \rightarrow X_{i} \rightarrow \cdots \rightarrow X_{-1} \rightarrow X_{0}=N \rightarrow X_{1} \rightarrow \cdots \rightarrow X_{j} \rightarrow \cdots
$$

is sectional. Hence $N$ lies on an $A_{\infty}^{\infty}$-section.

As a consequence we have

Theorem 1.6. Let $M$ be an indecomposable $\Lambda$-module.

(a) If $M$ is the middle of a short chain then $M$ is on a short cycle.

(b) If $\Lambda$ has no $A_{\infty}^{\infty}$-sectional chains, then $M$ is the middle of a short chain if and only if it is on a short cycle.

Proof. (a) Let $X \rightarrow^{f} M \rightarrow^{g} D \operatorname{Tr} X$ be a short chain. Consider the almost split sequence

$$
0 \rightarrow D \operatorname{Tr} X \stackrel{\left(\begin{array}{c}
\alpha_{1} \\
\vdots \\
\alpha_{t}
\end{array}\right)}{\longrightarrow} E_{1} \coprod \cdots \coprod E_{t} \stackrel{\left(\beta_{1}, \ldots, \beta_{t}\right)}{\longrightarrow} X \rightarrow 0,
$$

where the $E_{i}$ are indecomposable. Choose $i$ such that the composition $M \rightarrow g$ $D T r X \rightarrow^{\alpha_{i}} E_{i}$ is not zero. If $E_{i} \rightarrow^{\beta_{i}} X$ is a monomorphism, then also the composition $M \rightarrow^{g} D \operatorname{Tr} X \rightarrow^{\alpha_{i}} E_{i} \rightarrow^{\beta_{i}} X$ is nonzero, so that we get a short cycle $M \rightarrow \beta_{i} \alpha_{i} g X \rightarrow{ }^{f} M$ if $f$ is not an isomorphism and a short cycle $M \rightarrow \beta_{i} \alpha_{i} g$ $X \rightarrow{ }^{f \beta_{i} \alpha_{i} g f} M$ if $f$ is an isomorphism.

If $E_{i} \rightarrow^{\beta_{i}} X$ is an epimorphism then the composition $E_{i} \rightarrow^{\beta_{i}} X \rightarrow^{f} M$ is not zero, so that we get a short cycle $M \rightarrow^{\alpha_{i} g} E_{i} \rightarrow f \beta_{i} M$.

(b) This is a direct consequence of Proposition 1.5.

\section{MODULES DETERMINED BY THEIR COMPOSITION FACTORS}

It is interesting to find sufficient conditions for two indecomposable modules $M$ and $N$ with the same composition factors to be isomorphic. This is known to be the case if $M$ and $N$ are not the middle of a short chain [1]. Also it is true if one of the modules, $M$, is assumed to be directing, that is, $M$ does not lie on a cycle of nonzero nonisomorphisms between indecomposable modules [6]. It would be interesting to know if it is sufficient that $M$ is not the middle of a short chain, thus getting a common generalization of these two results.

As an application of the results in $\S 1$ we prove that this is the case when there are no $A_{\infty}^{\infty}$-sectional chains, in particular, for finite representation type. We also study the property of not being the middle of a short chain with respect to passing to factor rings.

We start with the following observation. 
Proposition 2.1. Let $M$ and $N$ be indecomposable $\Lambda$-modules having the same composition factors, and assume that $M$ is not the middle of a short chain. If $M$ is not isomorphic to $N$, then we have a short cycle $M \rightarrow N \rightarrow M$.

Proof. If $P_{1} \rightarrow P_{0} \rightarrow X \rightarrow 0$ is a minimal projective presentation for a $\Lambda$ module $X$, by [1, Theorem 1.4] we have the formula $\langle X, Y\rangle-\langle Y, D \operatorname{Tr} X\rangle=$ $\left\langle P_{0}, Y\right\rangle-\left\langle P_{1}, Y\right\rangle$ for any $\Lambda$-module $Y$. Here $\langle Y, V\rangle$ denotes the length of $(Y, V)$ as an $R$-module, when $\Lambda$ is an artin $R$-algebra. Let $M$ and $N$ be indecomposable $\Lambda$-modules with the same composition factors, and assume that $M$ is not the middle of a short chain. We then have $\langle X, M\rangle-\langle M, D \operatorname{Tr} X\rangle=$ $\langle X, N\rangle-\langle N, D \operatorname{Tr} X\rangle$. Letting $X=M$, we have $\langle M, D \operatorname{Tr} M\rangle=0$. Hence we get $\langle M, N\rangle \neq 0$, so we have a nonzero nonisomorphism $M \rightarrow N$ if $M \not N$.

Since $D M$ is also not the middle of a short chain in $\bmod \Lambda^{\text {op }}$ and $D M$ and $D N$ have the same composition factors, we get $\langle D M, D N\rangle \neq 0$, and hence $\langle N, M\rangle \neq 0$. So if $M \not N$ we then have a nonzero nonisomorphism $N \rightarrow M$. Hence we have a short cycle $M \rightarrow N \rightarrow M$.

We have the following consequence.

Corollary 2.2. Let $M$ and $N$ be indecomposable $\Lambda$-modules having the same composition factors.

(a) If $M$ does not lie on a short cycle then $M \simeq N$.

(b) If $M$ is directing then $M \simeq N$.

(c) If $M$ is not the middle of a short chain and $N$ is not on an $A_{\infty}^{\infty}$-sectional chain, then $M \simeq N$.

(d) If $M$ is not the middle of a short chain and $\Lambda$ is of finite representation type, then $M \simeq N$.

Proof. (a) is a direct consequence of Propositions 1.6(a) and 2.1. Parts (b) and (c) are direct consequences of Propositions 1.5 and 2.1. Part (d) follows from (c) since an algebra of finite representation type has no sectional cycle by [4].

We end this section with the following general result, which will be used in $\S 4$.

Proposition 2.3. Let $M$ be an indecomposable module over an artin algebra $\Lambda$, and let $I$ be an ideal in $\Lambda$ contained in the annihilator of $M$.

If $M$ is not the middle of a short chain in $\bmod \Lambda$, then $M$ is not the middle of a short chain in $\bmod (\Lambda / I)$.

Proof. Assume that $M$ is the middle of a short chain $X \rightarrow M \rightarrow D \operatorname{Tr}_{\Lambda / I} X$ in $\bmod (\Lambda / I)$, so that we have $\operatorname{Hom}_{\Lambda / I}(X, M) \neq 0$ and $\operatorname{Hom}_{\Lambda / I}\left(M, D \operatorname{Tr}_{\Lambda / I} X\right)$ $\neq 0$. Since $X$ is not a projective $\Lambda / I$-module, it is also not a projective $\Lambda$ module. Then we have an almost split sequence $0 \rightarrow D \operatorname{Tr} X \rightarrow E \rightarrow X \rightarrow 0$ in $\bmod \Lambda$. Consider the exact commutative diagram

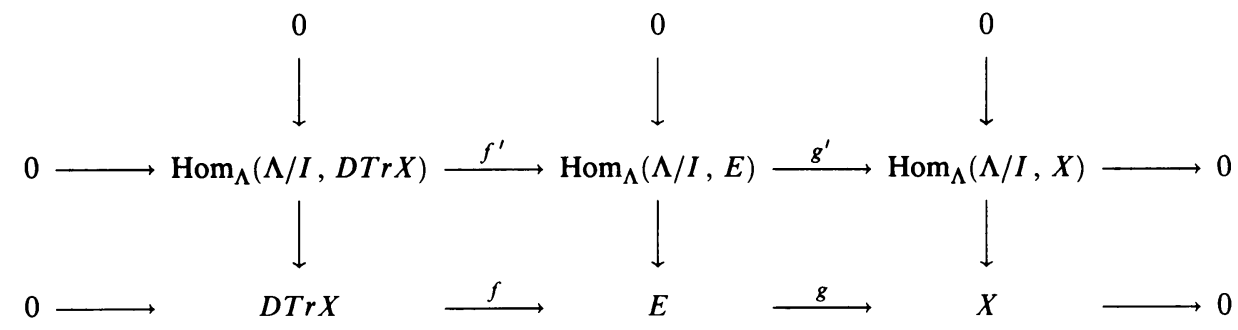


Since $g^{\prime}: \operatorname{Hom}_{\Lambda}(\Lambda / I, E) \rightarrow \operatorname{Hom}_{\Lambda}(\Lambda / I, X)$ is clearly right almost split in $\bmod (\Lambda / I)$, we have that $D \operatorname{Tr}_{\Lambda / I} X$ is a summand of $\operatorname{Hom}_{\Lambda}(\Lambda / I, D \operatorname{Tr} X)$ and hence is a submodule of $D \operatorname{Tr} X$. This shows that $\operatorname{Hom}_{\Lambda}(M, D \operatorname{Tr} X) \neq 0$, so that $M$ is the middle of a short chain in $\bmod \Lambda$.

\section{Sincere MOdUles that ARE NOT THE MidDle OF SHORT CHAINS}

It was shown by Ringel in [6] that if $M$ is an indecomposable sincere directing $\Lambda$-module then $\Lambda$ is a tilted algebra. This was generalized by Bakke in [2] as follows: If $M$ is a sincere $\Lambda$-module such that there is no chain of nonisomorphisms $M^{\prime} \rightarrow \cdots \rightarrow X \rightarrow \cdots \rightarrow \operatorname{Tr} D X \rightarrow \cdots \rightarrow M^{\prime \prime}$ between indecomposable modules with $M^{\prime}$ and $M^{\prime \prime}$ in add $M$, then $\Lambda$ is a tilted algebra. In particular, if we define an arbitrary $\Lambda$-module to be directing if there is no chain of nonisomorphisms $M^{\prime} \rightarrow \cdots \rightarrow M^{\prime \prime}$ between indecomposable modules with $M^{\prime}$ and $M^{\prime \prime}$ in add $M$, then Ringel's result holds also if $M$ is not indecomposable. It would be interesting to know whether the existence of a sincere $\Lambda$-module $M$ that is not the middle of a short chain implies that $\Lambda$ is a tilted algebra. In the next section we shall show that this is the case under the additional assumption that $M$ is indecomposable and $\Lambda$ is of finite representation type.

In this section we prove some general properties about $\Lambda$ when there is a sincere $\Lambda$-module that is not the middle of a short chain, for example, that $\Lambda$ has no oriented cycles in its ordinary quiver and has global dimension at most two.

For the first preliminary result, it is useful to view a $\Lambda$-module as a contravariant functor from add $\Lambda$ to abelian groups.

Proposition 3.1. Let $\Lambda$ be an artin algebra and $M$ a $\Lambda$-module that is not the middle of a short chain. For any nonzero map $f: P \rightarrow Q$ between indecomposable projective $\Lambda$-modules $P$ and $Q$, we have that $\operatorname{Hom}(f, M): \operatorname{Hom}_{\Lambda}(Q, M)$ $\rightarrow \operatorname{Hom}_{\Lambda}(P, M)$ is either a monomorphism or an epimorphism.

Proof. The proof can be found in the proof of $2.4\left(7^{\prime}\right)$ in [6], but for the convenience of the reader we include the argument here.

Assume that there is some nonzero map $f: P \rightarrow Q$ between indecomposable projective $\Lambda$-modules such that $\operatorname{Hom}(f, M)$ is neither a monomorphism nor an epimorphism. Let $X=\operatorname{Coker} f$. Since $\operatorname{Hom}(f, M)$ is not a monomorphism, $\operatorname{Hom}_{\Lambda}(X, M) \neq 0$. For $Y$ a finitely generated projective module, the natural map $D \operatorname{Hom}_{\Lambda}(Y, M) \rightarrow \operatorname{Hom}_{\Lambda}\left(Y^{*}, D M\right)$ is an isomorphism where $Y^{*}=\operatorname{Hom}_{\Lambda}(Y, \Lambda)$. It is easy to see that we then have a commutative diagram

$$
\begin{array}{cc}
D \operatorname{Hom}_{\Lambda}(P, M) \stackrel{D \operatorname{Hom}(f, M)}{\longrightarrow} D \operatorname{Hom}_{\Lambda}(Q, M) \\
\qquad \\
\operatorname{Hom}_{\Lambda}\left(P^{*}, D M\right) \stackrel{\operatorname{Hom}\left(f^{*}, D M\right)}{\longrightarrow} \operatorname{Hom}_{\Lambda}\left(Q^{*}, D M\right)
\end{array}
$$

where $f^{*}=\operatorname{Hom}(f, \Lambda)$. Since $\operatorname{Hom}(f, M)$ is not an epimorphism, $D \operatorname{Hom}(f, M)$ is not a monomorphism and, therefore, $\operatorname{Hom}\left(f^{*}, D M\right)$ is not a monomorphism. This implies that $\operatorname{Hom}_{\Lambda^{\circ \mathrm{p}}}\left(\operatorname{Coker} f^{*}, D M\right) \neq 0$. Since clearly Coker $f^{*} \simeq \operatorname{Tr} X$, we get $\operatorname{Hom}_{\Lambda^{\text {op }}}(\operatorname{Tr} X, D M) \neq 0$ and, consequently,

$$
\operatorname{Hom}_{\Lambda}(M, D T r X) \neq 0 \text {. }
$$


We have already seen that $\operatorname{Hom}_{\Lambda}(X, M) \neq 0$. Since $X$ is indecomposable because $Q$ is, we get that $M$ is the middle of a short chain.

As a consequence of Proposition 3.1 we get the following relationship between sincere and faithful modules (see also [6, 2.4( $\left.\left.7^{\prime}\right)\right]$ ). Note that a faithful module is always sincere.

Corollary 3.2. If $M$ is a sincere $\Lambda$-module that is not the middle of a short chain then $M$ is faithful.

Proof. Let $M$ be a sincere $\Lambda$-module that is not the middle of a short chain. Write $1=e_{1}+\cdots+e_{n}$, where the $e_{i}$ are primitive orthogonal idempotents. If ann $M \neq(0)$, there are some $i, j$ with $e_{i}($ ann $M) e_{j} \neq 0$. Choose $\lambda \in$ ann $M$ such that $e_{i} \lambda e_{j} \neq 0$. Then $\lambda$ induces by right multiplication a nonzero homomorphism $\mu: \Lambda e_{i} \rightarrow \Lambda e_{j}$. Since $M$ is sincere, $\operatorname{Hom}_{\Lambda}\left(\Lambda e_{i}, M\right) \neq 0$ and $\operatorname{Hom}_{\Lambda}\left(\Lambda e_{j}, M\right) \neq 0$. By the choice of $\mu$ it is easy to see that $\operatorname{Hom}(\mu, M)=0$ and hence, $\operatorname{Hom}(\mu, M)$ is neither a monomorphism nor an epimorphism. This gives a contradiction to Proposition 3.1, so that $M$ is faithful.

As a consequence we get the following information on $\Lambda$.

Corollary 3.3. Let $M$ be a sincere $\Lambda$-module that is not the middle of a short chain. Then the ordinary quiver of $\Lambda$ has no oriented cycle.

Proof. Assume that we have a cycle of nonzero nonisomorphisms $P_{1} \rightarrow f_{1} P_{2} \rightarrow f_{2}$ $\cdots \rightarrow P_{n} \rightarrow^{f_{n}} P_{n+1}=P_{1}$, where the $P_{i}$ are indecomposable projectives. By Proposition 3.1, $\operatorname{Hom}\left(f_{i}, M\right): \operatorname{Hom}\left(P_{i+1}, M\right) \rightarrow \operatorname{Hom}\left(P_{i}, M\right)$ is either a monomorphism or an epimorphism for each $i=1, \ldots, n$. Since $f=$ $f_{n} \cdots f_{2} f_{1}: P_{1} \rightarrow P_{1}$ is not an isomorphism and $P_{1}$ is indecomposable, $f$ is nilpotent and hence, $\operatorname{Hom}(f, M): \operatorname{Hom}_{\Lambda}\left(P_{1}, M\right) \rightarrow \operatorname{Hom}_{\Lambda}\left(P_{1}, M\right)$ is nilpotent. Then there must be both a proper epimorphism and a proper monomorphism amongst the $\operatorname{Hom}\left(f_{i}, M\right)$. So choose $i$ such that $\operatorname{Hom}\left(f_{i}, M\right)$ is a proper monomorphism, and let $j$ be such that $\operatorname{Hom}\left(f_{t}, M\right)$ is a monomorphism for $i \leq t<j$ and $\operatorname{Hom}\left(f_{j}, M\right)$ is a proper epimorphism. Here we define $f_{j+n}=f_{j}$ for $j=1, \ldots, n$. Then the composition $\operatorname{Hom}\left(f_{i}, M\right) \cdots \operatorname{Hom}\left(f_{j}, M\right)$ is nonzero and is neither an epimorphism nor a monomorphism, contradicting Proposition 3.1 .

We next want to investigate the global dimension of $\Lambda$; the following result is useful.

Lemma 3.4. Let $M$ be a sincere $\Lambda$-module that is not the middle of a short chain, and let $X$ be an indecomposable $\Lambda$-module.

(a) If $\operatorname{Hom}_{\Lambda}(M, X) \neq 0$ or $\operatorname{Hom}_{\Lambda}(\operatorname{Tr} D X, M)=0$, then $\operatorname{id}_{\Lambda} X \leq 1$.

(b) If $\operatorname{Hom}_{\Lambda}(X, M) \neq 0$ or $\operatorname{Hom}_{\Lambda}(M, D T r X)=0$, then $\operatorname{pd}_{\Lambda} X \leq 1$.

Proof. Let $M$ be a sincere $\Lambda$-module that is not the middle of a short chain. Let $X$ be indecomposable and assume first $\operatorname{Hom}_{\Lambda}(\operatorname{Tr} D X, M)=0$. Let $0 \rightarrow X \rightarrow I_{0} \rightarrow^{g} I_{1} \rightarrow \ldots$ be a minimal injective resolution of $X$. From [1, Theorem 1.4] we have the formula $\langle Y, X\rangle-\langle\operatorname{Tr} D X, Y\rangle=\left\langle Y, I_{0}\right\rangle-\left\langle Y, I_{1}\right\rangle$, for a $\Lambda$-module $Y$, where $\langle A, B\rangle$ denotes the length of $\operatorname{Hom}_{\Lambda}(A, B)$ as a module over $R$. Since $\langle\operatorname{Tr} D X, M\rangle=0$, we have $\langle M, X\rangle-\left\langle M, I_{0}\right\rangle+\left\langle M, I_{1}\right\rangle=0$. Because the sequence of $R$-modules $0 \rightarrow \operatorname{Hom}_{\Lambda}(M, X) \rightarrow \operatorname{Hom}_{\Lambda}\left(M, I_{0}\right) \rightarrow^{h}$ 
$\operatorname{Hom}_{\Lambda}\left(M, I_{1}\right)$ is exact, we see that $h$ must then be surjective by considering lengths. $M$ is faithful by Proposition 3.2, so we have an epimorphism $f: M^{n} \rightarrow I_{1}$ for some $n$. There is then some $t: M^{n} \rightarrow I_{0}$ with $g t=f$. Hence $g: I_{0} \rightarrow I_{1}$ is an epimorphism, so that $\operatorname{id}_{\Lambda} X \leq 1$.

If we assume $\operatorname{Hom}_{\Lambda}(M, X) \neq 0$, we have $\operatorname{Hom}_{\Lambda}(\operatorname{Tr} D X, M)=0$ since $M$ is not the middle of a short chain, so $\mathrm{id}_{\Lambda} X \leq 1$ by the first part. This finishes the proof of (a); (b) follows from (a) by duality.

Theorem 3.5. Let $\Lambda$ be an artin algebra, and assume that there is a sincere $\Lambda$-module $M$ that is not the middle of a short chain. Then $\operatorname{gl} \operatorname{dim} \Lambda \leq 2$.

Proof. Assume there is a sincere $\Lambda$-module $M$ that is not the middle of a short chain. Let $X$ be an indecomposable $\Lambda$-module that is a factor module of an injective module $I$. Since $M$ is sincere and not the middle of a short chain, $M$ is faithful by Corollary 3.2. Hence there is an epimorphism $M^{n} \rightarrow I$ for some $n>0$, so that $\operatorname{Hom}_{\Lambda}(M, X) \neq 0$. It follows that $\operatorname{id}_{\Lambda} X \leq 1$ by Lemma 3.4. This shows that $\operatorname{gl} \operatorname{dim} \Lambda \leq 2$.

The following gives some information on the modules of projective dimension two and injective dimension two.

Proposition 3.6. Let $\Lambda$ be an artin algebra with a sincere $\Lambda$-module $M$ that is not the middle of a short chain. Then no indecomposable $\Lambda$-module $X$ has both injective and projective dimension equal to two.

Proof. Assume $\Lambda$ has a sincere module $M$ that is not the middle of a short chain. Let $X$ be an indecomposable module of injective dimension two. Then $\operatorname{Hom}_{\Lambda}(M, X)=0$ and $\operatorname{Hom}(\operatorname{Tr} D X, M) \neq 0$ by Lemma 3.4(a), so that $\operatorname{pd}_{\Lambda} \operatorname{Tr} D X \leq 1$ by Lemma $3.4(\mathrm{~b})$.

Consider the almost split sequence $0 \rightarrow X \rightarrow E \rightarrow \operatorname{Tr} D X \rightarrow 0$, and let $Y$ be an indecomposable summand of $E$. Since $g l \operatorname{dim} \Lambda \leq 2$, we have that $\operatorname{Ext}_{\Lambda}^{2}(, \Lambda / \mathfrak{r})$ is right exact where $\mathfrak{r}=\operatorname{rad} \Lambda$. If $Y \rightarrow \operatorname{Tr} D X$ is a monomorphism, then it follows from the fact that $\operatorname{pd}_{\Lambda} \operatorname{Tr} D X \leq 1$ that $\operatorname{pd}_{\Lambda} Y \leq 1$. If $Y \rightarrow \operatorname{Tr} D X$ is an epimorphism, we have $\operatorname{Hom}_{\Lambda}(Y, M) \neq 0$ since $\operatorname{Hom}(\operatorname{Tr} D X, M) \neq 0$. Then $\operatorname{pd}_{\Lambda} Y \leq 1$ by Lemma $3.4(\mathrm{~b})$, so that $\operatorname{pd}_{\Lambda} E \leq 1$ and, consequently, $\operatorname{pd}_{\Lambda} X \leq 1$.

We next show the following connection between factor rings and endomorphism rings.

Proposition 3.7. Let $\Lambda$ be a basic artin algebra and $M$ a $\Lambda$-module that is not the middle of a short chain. Write $\Lambda=P \amalg Q$, where the simple summands of $P / \mathfrak{r} P$ are exactly the composition factors of $M$. Denote by $\tau_{Q}(\Lambda)$ the ideal of $\Lambda$ generated by all images of all maps from $Q$ to $\Lambda$. Then $\operatorname{End}_{\Lambda}(P)^{\text {op }} \simeq \Lambda / \tau_{Q}(\Lambda)$ and $\tau_{Q}(\Lambda)=$ ann $M$.

Proof. With the above assumptions and notations, consider the natural functor $F:$ add $P \rightarrow \operatorname{add}\left(\Lambda / \tau_{Q}(\Lambda)\right)$ defined by $F(P)=P / \tau_{Q}(P)$ on objects and defined naturally on morphisms. Let $f: P_{1} \rightarrow P_{2}$ be a nonzero map with $P_{1}$ and $P_{2}$ indecomposable and $F(f)=0$. Then the image of $f$ is in $\tau_{Q}\left(P_{2}\right)$ and we have that $f$ is the composition $P_{1} \rightarrow Q^{\prime} \rightarrow P_{2}$ with $Q^{\prime}$ in add $Q$.

Since $\left(Q^{\prime}, M\right)=0$, the composition $\left(P_{2}, M\right) \rightarrow\left(Q^{\prime}, M\right) \rightarrow\left(P_{1}, M\right)$ is zero, so $(f, M)$ is zero. Because $\left(P_{1}, M\right)$ and $\left(P_{2}, M\right)$ are not zero, $(f, M)$ 
is neither a monomorphism nor an epimorphism, which contradicts Proposition 3.1 since $M$ is not the middle of a short chain. Now obviously $F$ is full and dense, hence this shows that $F$ is an equivalence of categories, and consequently, $\operatorname{End}_{\Lambda}(P)^{\mathrm{op}} \simeq \Lambda / \tau_{Q}(\Lambda)$.

It is further clear that $\tau_{Q}(\Lambda)$ is contained in ann $M$. Since $M$ is not the middle of a short chain in $\bmod \Lambda$, it follows from Proposition 2.3 that $M$ is not the middle of a short chain in $\bmod \left(\Lambda / \tau_{Q}(\Lambda)\right)$. Since $\operatorname{Hom}_{\Lambda}\left(P^{\prime}, M\right) \neq$ 0 for each indecomposable summand $P^{\prime}$ of $P$, we see that $M$ is a sincere $\Lambda / \tau_{Q}(\Lambda)$-module. Then $M$ is faithful by Corollary 3.2 , so that $\operatorname{ann}_{\Lambda} M=$ $\tau_{Q}(\Lambda)$. This finishes the proof.

\section{TILTED ALGEBRAS OF FINITE REPRESENTATION TYPE}

In this section we show that if there is a sincere indecomposable $\Lambda$-module $M$ that is not the middle of a short chain and $\Lambda$ is in addition of finite representation type, then $\Lambda$ is a tilted algebra. For this the following preliminary results are useful.

Lemma 4.1. Let $X$ be $a \Lambda$-module and $A$ an indecomposable $\Lambda$-module. Assume there is a proper chain

$$
A_{n} \rightarrow \cdots \rightarrow A_{1} \rightarrow A_{0}=A
$$

of irreducible maps between indecomposable modules satisfying $\operatorname{Hom}_{\Lambda}\left(X, A_{n}\right) \neq$ 0 and $\operatorname{rad}\left(X, A_{j}\right)=0$ for $j<n$, and assume that this chain is chosen to be of minimal length with this property. If $A_{j}$ is not injective for $j=1, \ldots, n$ then (*) is sectional.

Proof. Let $X$ be a $\Lambda$-module and assume that we have a proper chain of irreducible maps $A_{n} \rightarrow \cdots \rightarrow A_{1} \rightarrow A_{0}=A$ between indecomposable modules, which is of minimal length with $n \geq 1, \operatorname{Hom}_{\Lambda}\left(X, A_{n}\right) \neq 0$ and $\operatorname{rad}\left(X, A_{i}\right)=0$ for $i<n$. Assume that no $A_{i}$ with $1 \leq i \leq n$ is injective. We want to show that the chain is sectional, so we can clearly assume that $n \geq 2$. Consider the almost split sequence $0 \rightarrow A_{n} \rightarrow E \rightarrow \operatorname{Tr} D A_{n} \rightarrow 0$. Then $\operatorname{rad}(X, B) \neq 0$ for some indecomposable summand $B$ of $E$, and we have a chain of irreducible maps $B \rightarrow \operatorname{Tr} D A_{n} \rightarrow \cdots \rightarrow \operatorname{Tr} D A_{2}$ between indecomposable modules. If the original chain was not sectional, we would have $\operatorname{Tr} D A_{j} \simeq A_{j-2}$ for some $j$ where $2 \leq j \leq n$. Then we get a contradiction by considering the chain $B \rightarrow \operatorname{Tr} D A_{n} \rightarrow \cdots \rightarrow \operatorname{Tr} D A_{j} \rightarrow A_{j-3} \rightarrow \cdots \rightarrow A_{0}=A$, which is shorter than the original one.

Lemma 4.2. Let $\Lambda$ be an artin algebra and $C$ an indecomposable $\Lambda$-module. Assume that there is a proper chain of irreducible maps between indecomposable modules from some indecomposable injective module to $C$. Then there is an indecomposable injective $\Lambda$-module I and an indecomposable $\Lambda$-module $Z$ such that $\operatorname{rad}(I, Z) \neq 0$ and $\operatorname{Hom}_{\Lambda}(Z, C) \neq 0$.

Proof. If $\operatorname{rad}(D \Lambda, C) \neq 0$, then we have $\operatorname{rad}(I, C) \neq 0$ for some indecomposable injective $\Lambda$-module $I$. Then we can choose $Z=C$.

Assume now that $\operatorname{rad}(D \Lambda, C)=0$. By assumption, we have a proper chain of irreducible maps $J \rightarrow \cdots \rightarrow C$ where $J$ is injective. Since $\operatorname{Hom}_{\Lambda}(D \Lambda, J) \neq$ 0 , by Lemma 4.1, there is a minimal proper chain $Z=A_{n} \rightarrow \cdots \rightarrow A_{1} \rightarrow A_{0}=$ $C$ of irreducible maps between indecomposable modules, with $\operatorname{Hom}_{\Lambda}(D \Lambda, Z)$ 
$\neq 0$ and $\operatorname{rad}\left(D \Lambda, A_{i}\right)=0$ for $i<n$. If $A_{i}$ was injective for some $i=$ $1, \ldots, n$, we would have $\operatorname{rad}\left(D \Lambda, A_{i-1}\right) \neq 0$, which is a contradiction. Hence the chain is sectional by Lemma 4.1 and, therefore, $\operatorname{rad}(Z, C) \neq 0$ by [5]. Since $\operatorname{Hom}_{\Lambda}(D \Lambda, Z) \neq 0$, there is some indecomposable injective module $I$ with $\operatorname{Hom}_{\Lambda}(I, Z) \neq 0$. Because $Z$ is not injective, it then follows that $\operatorname{rad}(I, Z) \neq$ 0 .

Lemma 4.3. let $\Lambda$ be an artin algebra of finite representation type having an oriented cycle of irreducible maps between indecomposable modules.

(a) There is a proper chain of irreducible maps between indecomposable modules $J=A_{n} \rightarrow A_{n-1} \rightarrow \cdots A_{1} \rightarrow A_{0}=P$, where $J$ is injective and $P$ is projective.

(b) There are indecomposable modules $I, P$, and $Z$, where $I$ is injective and $P$ is projective, and nonzero maps $f: I \rightarrow Z$ and $g: Z \rightarrow P$ with both $f$ and $g$ nonisomorphisms.

Proof. (a) Assume $\Lambda$ is an artin algebra of finite representation type. For a cycle $p$ of irreducible maps between indecomposable modules, define the distance $\delta(\mathscr{P}, p)$ from a projective module to $p$ to be the shortest length of chains of irreducible maps between indecomposable modules from a projective module to $p$, counting the number of morphisms. Since $\Lambda$ is of finite type, there is always some such chain. $\delta(\mathscr{P}, p)=0$ then means that the cycle contains some projective module. Similarly we define $\delta(p, \mathcal{J})$ to be the shortest length of chains of irreducible maps from $p$ to an injective module. We define $\delta(p)=$ $\delta(\mathscr{P}, p)+\delta(p, \mathcal{J})$ and want to show that if there is a cycle of irreducible maps between indecomposable modules then there is a cycle $p$ with $\delta(p)=0$, that is, containing both a projective and an injective module.

Assume that $p$ is a cycle of irreducible maps between indecomposable modules with $\delta(p)=d$ minimal, and assume $d>0$. By duality we may assume that $\delta(\mathscr{P}, p)=r>0$. Let $X_{0} \rightarrow X_{1} \rightarrow \cdots \rightarrow X_{n}=X_{0}$ be the cycle $p$, and let $P=Y_{r} \rightarrow \cdots \rightarrow Y_{1} \rightarrow Y_{0}=X_{t}$ be a chain of irreducible maps of length $r$ and with $P$ projective. We can clearly assume $t=0$. By [4] we know that the cycle $p$ is not sectional, that is, there is some $i$ with $X_{i} \simeq D \operatorname{Tr} X_{i+2}$ (where addition is modulo $n$ ). Since no $X_{j}$ is projective, we then have an oriented cycle $D \operatorname{Tr} X_{0} \rightarrow D \operatorname{Tr} X_{1} \rightarrow \cdots \rightarrow D \operatorname{Tr} X_{n}=D \operatorname{Tr} X_{0}$ and, consequently, an oriented cycle $p^{\prime}: X_{0} \rightarrow \cdots \rightarrow X_{i} \simeq D \operatorname{Tr} X_{i+2} \rightarrow \cdots \rightarrow D \operatorname{Tr} X_{0} \rightarrow Y_{1} \rightarrow$ $X_{0} \rightarrow X_{1} \rightarrow \cdots \rightarrow X_{i} \rightarrow X_{i+1} \rightarrow \cdots \rightarrow X_{n}=X_{0}$, with $\delta\left(\mathscr{P}, p^{\prime}\right)<r$. Clearly $\delta\left(p^{\prime}, \mathcal{J}\right) \leq \delta(p, \mathcal{J})$ and hence, $\delta\left(p^{\prime}\right)<\delta(p)$. This gives a contradiction and hence, we get a chain containing both an indecomposable projective and an indecomposable injective module. This gives a proper chain of irreducible maps between indecomposable modules from an indecomposable injective module $J$ to an indecomposable projective module $P$, where we may have $J=P$.

(b) By Lemma 4.2 and (a) there is some indecomposable injective $\Lambda$ module $I$ and an indecomposable $\Lambda$-module $Z$ such that $\operatorname{rad}(I, Z) \neq 0$ and $\operatorname{Hom}_{\Lambda}(Z, P) \neq 0$. If $\operatorname{rad}(Z, P)=0$, we have $Z \simeq P$. Then we can replace $Z$ by some indecomposable summand of $\mathfrak{r} P$.

We now have the following main result.

Theorem 4.4. Let $\Lambda$ be an artin algebra of finite representation type, and assume that there is some sincere indecomposable $\Lambda$-module $M$ that is not the middle 
of a short chain. Then $M$ is directing and, consequently, $\Lambda$ is a tilted algebra.

Proof. Let $\Lambda$ be an artin algebra of finite representation type having an indecomposable sincere module $M$ that is not the middle of a short chain. Assume that there is some oriented cycle of irreducible maps between indecomposable modules in $\bmod \Lambda$. Then, by Lemma 4.3, there are indecomposable modules $P, I$, and $Z$, with $P$ projective and $I$ injective, and nonzero maps $f: I \rightarrow Z$ and $g: Z \rightarrow P$ that are not isomorphisms. Since $M$ is sincere, $M$ is faithful by Corollary 3.2. Hence we have a monomorphism $\Lambda \rightarrow M^{n}$ and an epimorphism $M^{t} \rightarrow D \Lambda$ for some $n>0$ and $t>0$. Then there are induced nonzero nonisomorphisms $M \rightarrow D \Lambda \rightarrow I \rightarrow Z$ and $Z \rightarrow P \rightarrow \Lambda \rightarrow M$, so that $M$ is on a short cycle. Since $\Lambda$ is of finite representation type, $M$ must then be the middle of a short chain, which is a contradiction. Hence there are no oriented cycles of irreducible maps between indecomposable modules in $\bmod \Lambda$, so that all indecomposable $\Lambda$-modules are directing. Finally we use that the existence of an indecomposable sincere directing module implies that $\Lambda$ is a tilted algebra [6] (see also [2]).

We end the section with an application to modules determined by their top and socle. Bakke and Smalø have shown that if $M$ and $N$ are indecomposable $\Lambda$-modules with $\operatorname{Soc} M \simeq \operatorname{Soc} N$ and $M / \mathfrak{r} M \simeq N / \mathfrak{r} N$, then $M \simeq N$ if $M$ is assumed to be directing [3]. We have the following generalization in the case of finite representation type, as an application of Theorem 4.3. This hypothesis can be dropped if it can be dropped in Theorem 4.3.

Proposition 4.5. Let $\Lambda$ be an artin algebra of finite representation type. If $M$ and $N$ are indecomposable $\Lambda$-modules with $\operatorname{Soc} M \simeq \operatorname{Soc} N$ and $M / \mathfrak{r} M \simeq$ $N / \mathfrak{r} N$ and if $M$ is not the middle of a short chain, then $M \simeq N$.

Proof. Assume $\Lambda$ is an artin algebra of finite type and that $M$ is an indecomposable $\Lambda$-module that is not the middle of a short chain. Let $P$ be the direct sum of one copy of each of the indecomposable projective modules having a nonzero map to $M$, and let $\Gamma=\operatorname{End}_{\Lambda}(P)^{\text {op }}$. Let $\mathscr{J}$ be the full subcategory of $\bmod \Lambda$ whose objects are the $X$ in $\bmod \Lambda$ such that the projective cover of $\operatorname{Soc} X \amalg X / \mathfrak{r} X$ is in add $P$. Then we have an equivalence of categories $(P):, \mathscr{J} \rightarrow \bmod \Gamma$ (see [1]). Now assume $N$ is an indecomposable $\Lambda$-module with $\operatorname{Soc} N \simeq \operatorname{Soc} M$ and $N / \mathfrak{r} N \simeq M / \mathfrak{r} M$. Clearly $M, N$, $\operatorname{Soc} M$, and $M / \mathfrak{r} M$ are in $\mathscr{F}$, and it is easy to see that

$$
\operatorname{Soc}(P, M) \simeq(P, \operatorname{Soc} M) \simeq(P, \operatorname{Soc} N) \simeq \operatorname{Soc}(P, N)
$$

and

$$
(P, M) / \operatorname{rad}(P, M) \simeq(P, M / \mathfrak{r} M) \simeq(P, N / \mathfrak{r} N) \simeq(P, N) / \operatorname{rad}(P, N) .
$$

Since $M$ is not the middle of a short chain in $\bmod \Lambda$, it follows from Proposition 3.7 that $\Gamma \simeq \Lambda / \operatorname{ann} M$. Hence $M$ is not the middle of a short chain over $\Lambda$ /ann $M$ by Proposition 2.3 , that is, $(P, M)$ is not the middle of a short chain in $\bmod \Gamma$. Since $(P, M)$ is a sincere $\Gamma$-module, it follows from Theorem 4.3 that $(P, M)$ is directing. Because $(P, M)$ and $(P, N)$ have isomorphic socles and tops, $(P, M)$ and $(P, N)$ must be isomorphic by [3]. Since $M$ and $N$ are both in $\mathscr{J}$, we conclude that $M$ and $N$ are isomorphic $\Lambda$-modules. 


\section{REFERENCES}

1. M. Auslander and I. Reiten, Modules determined by their composition factors, Illinois J. Math. 29 (1985), 280-301.

2. Ø. Bakke, Some characterizations of tilted algebras, Math. Scand. 63 (1988), 43-50.

3. Ø. Bakke and S. O. Smalø, Modules with the same socles and tops as directing module are isomorphic, Comm. Algebra 15 (1987), 1-9.

4. R. Bautista and S. O. Smalø, Nonexistent cycles, Comm. Algebra 11 (1983), 1755-1767.

5. K. Bongartz, On a result of Bautista and Smalo on cycles, Comm. Algebra 11 (1983), 21232124.

6. C. M. Ringel, Tame algebras and integral quadratic forms, Lecture Notes in Math., vol. 1099, Springer-Verlag, Berlin and New York, 1984.

Department of Mathematics and Statistics, University of Trondheim, AVH, N-7055, DragVoll, NorwaY

E-mail address: Idun.Reiten@avh.unit.no

Institute of Mathematics, Nicholas Copernicus University, Chopina 12/18, 87-100 TORUŃ, POLAND

E-mail address: Skowron@pltumk11.bitnet

Department of Mathematics and Statistics, University of Trondheim, AVH, N-7055, DRAGVOLL, Norway

E-mail address: Sverre.Smalo@avh.unit.no 